\title{
NOVEL PERSPECTIVES IN THE MONITORING OF TRANSPORT INFRASTRUCTURES BY SENTINEL-1 AND COSMO-SKYMED MULTI-TEMPORAL SAR INTERFEROMETRY
}

\author{
Valerio Gagliardi ${ }^{\mathrm{a}}$, Luca Bianchini Ciampoli $^{\mathrm{a}}$, Fabrizio D'Amico $^{\mathrm{a}}$, Amir M. Alani $^{\mathrm{b}}$, Fabio Tosti $^{\mathrm{b}}$, \\ Maria Libera Battagliere ${ }^{\mathrm{c}}$, Andrea Benedetto ${ }^{\mathrm{a}}$ \\ ${ }^{a}$ Department of Engineering, Roma Tre University, Via Vito Volterra 62, 00146, Rome, Italy \\ ${ }^{\mathrm{b}}$ School of Computing and Engineering, University of West London (UWL), St Mary's Road, Ealing, \\ London W5 5RF, UK \\ ${ }^{\mathrm{c}}$ Italian Space Agency (ASI), Via del Politecnico, 00133, Rome, Italy
}

\begin{abstract}
In recent years, successful applications of the Synthetic Aperture Radar Interferometry (InSAR) for the monitoring of subsidence and deformations in transport infrastructures have been reported in the literature. The main advantage of this technique compared to other non-destructive surveying methodologies is in the possibility to perform fast networklevel surveys as well as the provision of time-series of the displacements by multi-temporal data acquisitions. Given the medium ground resolution, C-band imagery are usually not employed for transport infrastructure monitoring as it is considered unlikely to obtain sufficiently accurate information. However, the use of medium resolution SAR data has not been thoroughly investigated until now and is still an open challenge. This study presents a novel approach for transport assets monitoring, based on the synergistic use of medium resolution (C-Band) and high resolution (X-Band) SAR imagery. To this effect, a multitemporal SAR Interferometry analysis of high and mediumresolution datasets is performed on a runway of the Leonardo Da Vinci Airport in Rome, Italy. The data were acquired by the Sentinel-1A and the COSMO-SkyMed missions, respectively. A comparison between the results from medium and high-resolution datasets demonstrates the viability of using multi-frequency SAR imagery, and pave the way to the development of new methodologies for the monitoring of transport infrastructures.
\end{abstract}

Index Terms - Remote Sensing, COSMO-SkyMed, Persistent Scatterers Interferometry, Non-Destructive Assessment, Transport Infrastructure Monitoring, InSAR, C-Band Sentinel 1, Satellite Remote Sensing.

\section{INTRODUCTION}

Monitoring the conditions of transport infrastructures, such as railways, roads and bridges, is a priority for asset owners and administrators to ensure structural stability, operational safety and to prevent damage and deterioration - leading to expensive rehabilitation - or even failures or collapses [1]. Currently, several on-site non-destructive testing (NDT) technologies and sensors are available for subsidence monitoring and displacement mapping. Amongst the others, accelerometers [2], strain gauges [3], Global Position System (GPS), levelling [4-5], Ground Penetrating Radar (GPR), Infrared Thermography (IRT) [6-8] and terrestrial SAR Interferometry [9], are recognised as viable technologies for infrastructure monitoring.

However, on-site surveys are costly and are difficult to implement at the network level due to economic and administrative budget constraints. To overcome this limitation, several innovative satellite-based remote sensing techniques, i.e., Persistent Scatterers Interferometry (PSI) among which the PS-InSAR [10-11] and the Small BAseline Subset (SBAS) [12], have gained momentum in the last few years for the monitoring of transport assets and the investigation of nearby areas.

\section{AIMS AND OBJECTIVES}

This research aims at demonstrating the potential of the medium-range resolution Synthetic Aperture Radar (SAR) imagery for transport asset monitoring and the effective detection of areas of concern.

The main objective is to prove the viability to use medium resolution C-Band data to identify features of interest requiring further investigation utilizing complementary higher-resolution X-Band SAR products. Within this context, this study reports a novel approach, based on the synergistic use of the medium resolution Sentinel 1-A (CBand) and high-resolution COSMO-SkyMed (X-Band) SAR imagery to facilitate an effective management of linear transport infrastructures. 


\section{METHODOLOGY}

\subsection{Multi-Temporal InSAR for Transport Infrastructure Monitoring}

The working framework of the multi-temporal Interferometric Synthetic Aperture Radar (SAR) technique relies on a statistical analysis of the signals emitted by the on-satellite sensor and back-scattered by a network of coherent targets on the ground, i.e., the Persistent Scatterers (PS). This approach allows to estimate the displacements occurred between different acquisitions by a separation between the phase shift related to the ground motions and the phase component due to the atmosphere, the topography and the signal noise contributions $[11,12]$.

An advantage of these techniques is the relatively lighter data-processing required for the assessment of displacements and the detection of critical areas, as opposed to the higher computational load needed in other approaches. Therefore, the multi-temporal InSAR approach has proven to be ideal in monitoring transport infrastructure, as the high density of radar stable targets allows for more accurate measurements. To this effect, several scientific contributions related to this application of the PSI techniques can be found in the literature, as reported in Tab. 1 .

Tab. 1 -PSI applications for transport infrastructures monitoring

\begin{tabular}{cc}
\hline Infrastructure type & References \\
\hline Railways & {$[13-19]$} \\
Highways and tunnels & {$[20-22]$} \\
Bridges & {$[23-27]$} \\
Airport runways & {$[28-30]$} \\
\hline
\end{tabular}

This evidence confirms that the use of these satellite-based remote sensing techniques to these specific areas of endeavour is becoming a popular asset management tool.

\subsection{The Case Study}

\section{EXPERIMENTAL FRAMEWORK}

To achieve the above-set objectives, a dataset of SAR images from both the Sentinel 1A and COSMO-SkyMed missions covering the area of the Leonardo Da Vinci International Airport in Rome, Italy, was collected in the time interval 2017-2019, and processed by means of the PSI technique. Specifically, data in this study are referred to Runway 3. A comparison between the results obtained by the PS-analysis developed in different operative frequency was performed to assess the viability of using C-band data for the monitoring of airport runways. The potential of the proposed methodology for an integration with other on-site NDTs (e.g., GPR and Laser Scanner) was also investigated.

\subsection{SAR Datasets}

A multi-frequency SAR dataset of C-Band and X-Band data was collected and processed by the PSI technique to quantify and evaluate potential displacements on the inspected runway. The C-Band SAR products were acquired by the European Space Agency (ESA) in the framework of the Sentinel-1 mission. More specifically, the Sentinel-1 satellite, operates at a frequency of $5.4 \mathrm{GHz}$, which allows a high-frequent routine detection of displacements with a centimetre accuracy. In this analysis, 23 Single Look Complex (SLC) SAR products collected in the period from 2017 to 2019, were acquired and processed.

Furthermore, the assessment of the area is performed through the processing of higher resolution X-Band SAR images collected by the COSMO-SkyMed mission (COSMO-SkyMed Product - CASI: Italian Space Agency, 2017-2019, All Rights Reserved) delivered under the license to use. The COSMO-SkyMed system operates in Xband at a frequency of $9.6 \mathrm{GHz}$ corresponding to a wavelength of $3.1 \mathrm{~cm}$. This allows to collect data with ground-resolution cells sized $3 \times 3 \mathrm{~m}$ and to detect displacements with a millimetre accuracy, under ideal conditions.

\subsection{PSI Processing}

The SAR datasets were processed according to the PSI method [10] by means of the Interferometric Stacking Module of the Software SARscape [31] integrated in the Software ENVI, licensed within the framework of the European Space Agency (ESA) approved project "STRAIN2: Sensing TRAnsport INfrastructures 2" (EOhops proposal ID 53071). More specifically, the PSI technique operates by the application of consecutive stages $[10,11]$ : as a result, stable reflectors, i.e., the PSs, can be identified over the inspected area However, SAR satellites can only detect displacements in the Line-of-Sight (LoS), with reference to a specific orbit-related incident angle. a)

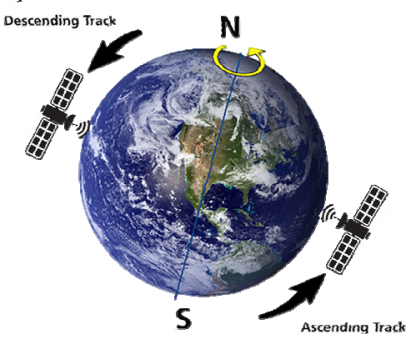

b)

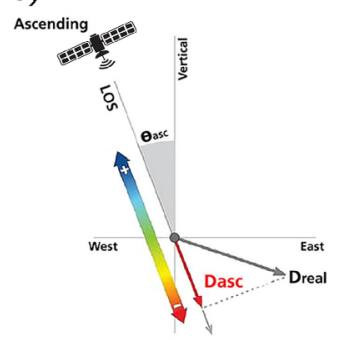

Figure 1: a) SAR data acquisition along an Ascending and a Descending orbit; b) Ascending acquisition geometry: the displacement $D_{\text {asc }}$ measured by the SAR is a component of the real displacement $D_{\text {real }}$ 
Therefore, the displacement detected is a component of the real displacement that occurred on the ground. Different approaches were proposed to evaluate the real displacements-velocity-vector, from datasets acquired in different acquisition geometries (i.e., Ascending and Descending) [32, 33]. For the investigated area of interest, SAR images in ascending acquisition geometries were used for both the Sentinel-1A and COSMO-SkyMed missions, in the observation period of this study. To this effect, in this work we refer only to the displacements detected in the LoS-direction of the SAR sensors.

\section{RESULTS}

With reference to the methodology described in section 2, the presented multi-temporal PSI technique was effective at detecting potential areas subject to an evolving subsidence and down-lifting displacements. In more detail, several PSs were identified over the inspected Runway 3 . The set of PSs resulting from the processing of the Sentinel-1 and COSMO-SkyMed data (ascending geometry) from 09/2017 to $01 / 2019$ is shown in Fig. $2(a, b)$.

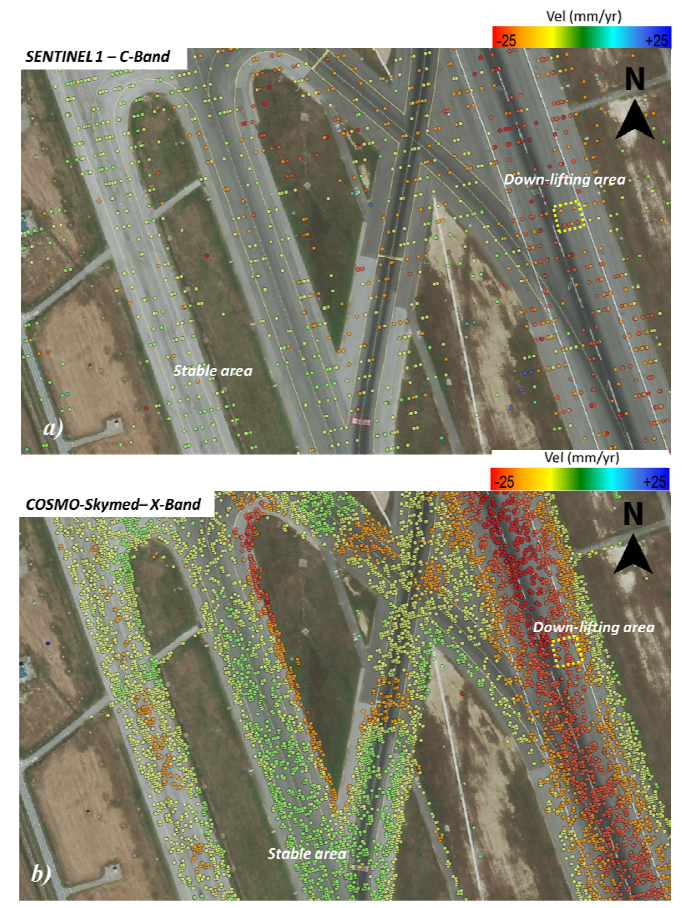

Figure 2: PSI results on the airport Runway 3 displayed in relation to the average trend of velocity in $\mathrm{mm} / \mathrm{yr}$ : a) PS outputs by the Sentinel-1A mission (ESA) and b): detailed results of the COSMO-SkyMed Product (CASI - Italian Space Agency)

Several PSs present a coherent deformation trend associated to a down-lifting occurrence, most likely related to subsidence caused by the low bearing capacity of the subgrade.
As expected, the processing of the X-Band SAR images allow to detect a larger amount of PS compared to the CBand sensors, due to the higher resolution. This can be observed in both Fig.1a and Fig.1b, where the identified PSs have been exported into a GIS platform and displayed in relation to the average velocity motion. The area marked in yellow in Fig. 2 shows a PS cluster characterized by a downlifting trend (e.g., subsidence) in both the SAR datasets. The average velocity value for this occurrence is $-14.9 \mathrm{~mm} / \mathrm{yr}$ for the Sentinel-1A dataset and about $-18.2 \mathrm{~mm} / \mathrm{yr}$ for the COSMO-SkyMed dataset. To compare quantitatively the trend of the two SAR datasets, an historical displacements analysis was conducted. To this effect a comparison between the values of displacement associated with the PSs identified in the sample area is reported in Fig. $3 \mathrm{a}, \mathrm{b}$. Historical time-series displacement (mm)

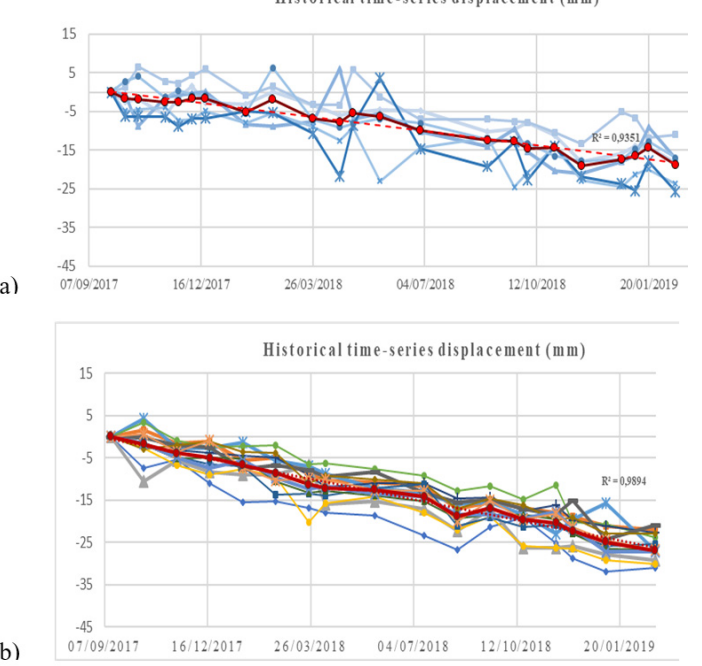

Figure 3. PSI time-series and deformation velocity from: a) PS samples related to the Sentinel 1A SAR images provided by ESA and b) PS samples related to the COSMO-SkyMed Product (CASI - Italian Space Agency 2017- 2019).

The maximum value of the relative down-lifting displacement is about $-25.0 \mathrm{~mm}$ for the Sentinel-1 data, and around $-30.0 \mathrm{~mm}$ for the COSMO-SkyMed. Both these datasets highlight the same down-lifting deformation trend. The $\mathrm{R}^{2}$ associated to the linear fitting of the average values of the PS displacements is calculated for each dataset. This value is observed to be high for the Sentinel-1A $\left(\mathrm{R}^{2}=0.93\right)$ dataset and the COSMO-SkyMed time-series $\left(\mathrm{R}^{2}=0.98\right)$. This indicates that the linear model might be considered as representative of the down-lifting deformation trend in the PS sample for both the C-Band and X-Band acquisitions. In addition, a statistical comparison between the two datasets of displacement velocities was conducted by means of a two-tailed t-test, with the alpha level for the $p$-value being assumed as 0.05 . The test returned a $p$-value of 0.0005 (i.e. $<0.05$ ), thereby indicating that the difference between the two samples is not statistically significant. This information is of interest for a potential integration of these outcomes 
with complementary on-site investigations (e.g., levelling or GPR) on critical areas identified preliminarily by the PSI technique. This approach could allow for a more effective planning of preventive maintenance on the runway, to limit full rehabilitation activities.

\section{CONCLUSIONS}

This research demonstrates the potential of the C-Band PSI remote-sensing technique as an innovative methodology for the monitoring of transport infrastructures. To this purpose, $\mathrm{X}$-Band COSMO-SkyMed products provided by the Italian Space Agency (ASI) and the Sentinel-1A missions provided by European Space Agency (ESA) were acquired and processed. A comparison between the results of data processed with the two resolutions demonstrates the viability of using multi-frequency SAR data, and sets novel perspectives in the monitoring of transport infrastructures. This information is fundamental to improve upon the capacity of pavement management systems (PMSs) to predict critical displacements and optimise maintenance interventions prior to pavement structural failure. Additionally, it is worth to note that for more accurate data the higher resolution X-band data must be adopted, as the high-resolution and the millimetre precision of the measurements allow to perform more accurate assessment procedures over the investigated infrastructure.

\section{ACKNOWLEDGMENTS}

The authors wish to thank the European Space Agency (ESA) for providing Sentinel-1 dataset for research purpose in this project. The COSMO-SkyMed Products ${ }^{\circledR}$ - CASI: Italian Space Agency, 2017-2019, All Rights Reserved- are (C) of the ASI (Italian Space Agency), delivered under the license to use. This research is supported by the Italian Ministry of Education, University and Research under the National Project "EXTRA TN", PRIN 2017, Prot. 20179BP4SM

\section{REFERENCES}

[1] P.C. Chang, A. Flatau \& S. C Liu. Review Paper: Health Monitoring of Civil Infrastructure. Structural Health Monitoring, 2(3), 257-267. 2003.

[2] C. Kongyang, L. Mingming, F. Xiaopeng, W. Mingming and Jinwu Wu, "Road condition monitoring using on-board Three-axis Accelerometer and GPS Sensor," 6th International ICST Conference on Communications and Networking in China, Harbin, 2011

[3] J. Olund, \& J. DeWolf, Passive Structural Health Monitoring of Connecticut's Bridge Infrastructure. Journal of Infrastructure Systems, 13(4), 330-339. 2007

[4] A. Mossop, P. Segall, Subsidence at The Geysers Geothermal Field, N. California from a comparison of GPS and leveling surveys. Geophys. Res. Lett., 24, 1839-1842, 1997

[5] H.P. Sato, K. Abe, O. Ootaki, GPS-measured land subsidence in Ojiya City, Niigata Prefecture, Japan. Eng. Geol., 67, 379-390, 2003.

[6] T. Saarenketo \& T. Scullion, (2000). Road evaluation with ground penetrating radar. Journal of Applied Geophysics, 43(2-4), 119-138.

[7] S. Lagüela, M. Solla, I. Puente, \& F. J. Prego, (2018). Joint use of GPR, IRT and TLS techniques for the integral damage detection in paving. Construction and Building Materials, 174, 749-760.

[8] L. Bianchini Ciampoli, S. S. Artagan, F. Tosti, V. Gagliardi, A. M Alani and Benedetto A., A comparative investigation of the effects of concrete sleepers on the GPR signal for the assessment of railway ballast, $17^{\text {th }}$ International Conference on Ground Penetrating Radar (GPR), Rapperswil, pp. 1-4, doi: 10.1109/ICGPR.2018.8441588, 2018.
[9] P. Mazzanti et al. (2015) Terrestrial SAR Interferometry Monitoring of Natural Slopes and Man-Made Structures. Engineering Geology for Society and Territory - Volume 5. Springer, Cham

[10] A. Ferretti, C. Prati, F. Rocca, Permanent scatters in SAR interferometry. IEEE Trans Geosci Remote Sens 39(1):8-20, 2001. https ://doi.org/10.1109/36.89866

[11] A. Ferretti, C. Prati, F. Rocca Nonlinear subsidence rate estimation using permanent scatterers in differential SAR interferometry. IEEE Trans Geosci Remote Sens 38(5):2202$2212,2000$.

[12] R. Lanari, O. Mora, M. Manunta, J.J. Mallorqui, P. Berardino, E. Sansosti, A small baseline approach for investigating deformation on full resolution differential SAR interferograms. IEEE Trans. Geosci. Remote Sens. 42, 1377-1386, 2004.

[13] L. Bianchini Ciampoli, V. Gagliardi, C. Clementini, et al. Transport Infrastructure Monitoring by InSAR and GPR Data Fusion. Surv Geophys 41, 371-394. 2020. https://doi.org/10.1007/s10712-019-09563-7

[14] L. Chang, R. P. Dollevoet, \& R. F. Hanssen, Nationwide Railway Monitoring Using Satellite SAR Interferometry. IEEE Journal of Selected Topics in Applied Earth Observations and Remote Sensing, 10(2),596-604.2017.

[15] F. D'Amico, V. Gagliardi, L. Bianchini Ciampoli, F. Tosti, Integration of InSAR and GPR Techniques for Monitoring Transition Areas in Railway Bridges. NDT\&E International. 2020.

[16] X. Qin, M. Liao, L. Zhang, \& M. Yang, Structural Health and Stability Assessment of High-Speed Railways via Thermal Dilation Mapping with Time-Series InSAR Analysis. IEEE Journal of Selected Topics in Applied Earth Observations and Remote Sensing, 10(6), 2999-3010. 2017.

[17] V. Gagliardi, L. Bianchini Ciampoli, F. D'Amico, A. M. Alani, F. Tosti, M. L. Battagliere, A. Benedetto, Bridge monitoring and assessment by high-resolution satellite remote sensing technologies, Proc. SPIE 11525, SPIE Future Sensing Technologies. 2020. doi: $10.1117 / 12.2579700$

[18] Yang Z., Schmid F., Roberts C., Assessment of railway performance by monitoring land subsidence. In: 6th IET conference on railway condition monitoring (RCM 2014), pp 1-6. $2014 \mathrm{https}: / /$ doi. org/10.1049/cp.2014.1000

[19] F. Tosti, V. Gagliardi, F. D'Amico, A.M. Alani, Transport infrastructure monitoring by data fusion of GPR and SAR imagery information. Transp Res Proc 2020; 45:771-778. 721. https://doi.org/10.1016/j.trpro.2020.02.097

[20] F. Koudogbo, A. Urdiroz, JG. Robles, G. Chapron, G. Lebon, V. Fluteaux, G. Priol Radar interferometry as an innovative solution for monitoring the construction of the Grand Paris Express metro network - first results. In: World tunnel conference, 2-25 April, Dubai, 2018

[21] G. Barla et al., InSAR monitoring of tunnel induced ground movements. Geomechanik und Tunnelbau 9(1):15-22, 2016

[22] D. Perissin, Z. Wang, \& H. Lin, (2012). Shanghai subway tunnels and highways monitoring through Cosmo-SkyMed Persistent Scatterers. ISPRS Journal of Photogrammetry and Remote Sensing, 73, 58-67.

[23] A. M. Alani, F. Tosti, L. Bianchini Ciampoli, V. Gagliardi, A. Benedetto, Integration of GPR and InSAR methods for the health monitoring of masonry arch bridges. NDT\&E International. 2020. https://doi.org/10.1016/j.ndteint.2020.102288

[24] V. Gagliardi, A. Benedetto, L. Bianchini Ciampoli, F. D'Amico, A. M. Alani, F. Tosti, Health monitoring approach for transport infrastructure and bridges by satellite remote sensing Persistent Scatterers Interferometry (PSI), Proc. SPIE 11534. 2020. https://doi.org/10.1117/12.2572395

[25] J. Jung, D-J Kim, SK Palanisamy Vadivel, S-H Yun. Long-term deflection monitoring for bridges using $\mathrm{X}$ and $\mathrm{C}$-band time-series SAR interferometry. Remote Sens 2019;11(11):1258.715

[26] L. Bianchini Ciampoli, V. Gagliardi, A. Calvi, F. D’Amico, F. Tosti, Automatic network-level bridge monitoring by integration of InSAR and GIS catalogues. Proceedings of SPIE 2019. https://doi.org/10.1117/12.2527299

[27] V. Gagliardi, L. Bianchini Ciampoli, F. D’Amico1, A. M. Alani, F. Tosti, A. Benedetto, Multi-Temporal SAR Interferometry for Structural Assessment of Bridges: The Rochester Bridge Case Study. International Airfield and Highway Pavements Conference 2021

[28] M. Gao, H. Gong, B. Chen, C. Zhou, W. Chen, Y. Liang, M. Shi, Y. Si, (2016). InSAR time-series investigation of long-term ground displacement at Beijing Capital International Airport, China.Tectonophysics, 691, 271-281.

[29] L. Bianchini Ciampoli; V. Gagliardi, C. Ferrante, A. Calvi, F. D’Amico, F. Tosti, Displacement Monitoring in Airport Runways by Persistent Scatterers SAR Interferometry. Remote Sens. (2020), 12, 3564. https://doi.org/10.3390/rs12213564

[30] L. Jiang, \& H. Lin, (2010). Integrated analysis of SAR interferometric and geological data for investigating long-term reclamation settlement of Chek Lap Kok Airport, Hong Kong. Engineering Geology, 110(3-4), 77-92.

[31] SARscape technical description, 2012. Internet resource accessed 2020-12-28 at http://www.sarmap.ch/pdf/SARscapeTechnical.pdf

[32] G. Dalla Via, M. Crosetto., and B. Crippa., Resolving vertical and east-west horizontal motion from differential interferometric synthetic aperture radar: The L'Aquila earthquake. Journal of geophysical research: solid earth 117, no. B2.2012.

[33] V. Gagliardi, L. Bianchini Ciampoli, F. D'Amico, F. Tosti, A. Alani and A. Benedetto. A Novel Geo-Statistical Approach for Transport Infrastructure Network Monitoring by Persistent Scatterer Interferometry (PSI). In: 2020 IEEE Radar Conference, Florence, Italy, 2020, pp. 1-6, doi: 10.1109/RadarConf2043947.2020.9266336 